\title{
Sandwich mixer-reactor: influence of the diffusion coefficient and flow rate ratios $\dagger$
}

\author{
Mélanie Abonnenc, Jacques Josserand and Hubert H. Girault*
}

\author{
Received 8th September 2008, Accepted 15th October 2008 \\ First published as an Advance Article on the web 17th November 2008 \\ DOI: $10.1039 / b 815581 j$
}

\begin{abstract}
A sandwich mixer consists of mixing two solutions in a channel, one central laminar flow being sandwiched between two outer flow solutions. The present numerical study considers the convectiondiffusion of two reacting species A and B, provided respectively by the two incoming solutions. The simulations show how the diffusion coefficient, flow rate and species concentration ratios influence, via the transversal diffusion length and reaction kinetics, the reaction extent at the end of the sandwich mixer. First, this extent can be enhanced up to $60 \%$ if the species with the lowest diffusion coefficient is located in the outer solutions where the flow velocity is small compared to that of the central part (higher residence time). Secondly, decreasing the outer flow rates (to confine the reaction close to the walls) and increasing the local concentration to keep the same flux ratio improve the extent by $300 \%$. Comparison with a bi-lamination passive mixer, with an ideal mixer and an electro-osmotic driven flow mixer is presented. These conclusions are also demonstrated for consecutive reactions, showing an amplification of the effects described above. The results are also presented versus the residence time in the mixer-reactor to show the time window for which the gain is appreciable.
\end{abstract}

\section{Introduction}

Microfluidics is now established in the field of (bio)chemistry and life sciences as a good alternative to all conventional laboratoryscale equipment, because it allows low reagent and time consumption, lower costs and high-throughput. ${ }^{1-3}$ Because of the small dimensions of the microchannels and the limited range of obtainable linear flow rates, flow in microchannels is generally confined to the laminar regime and mixing is dominated by molecular diffusion. Micromixing may be accomplished using different approaches. ${ }^{4,5}$ Active mixing may involve external energy sources such as electro-osmotic flow, ${ }^{6,7}$ external pressure gradient, ${ }^{8}$ electrokinetic instability ${ }^{9,10}$ or shaken droplets ${ }^{11}$ to perturb fluid streamlines to induce a macroscopic mixing. Another approach may consist in passive micromixing ${ }^{\mathbf{1 2}}$ that relies mainly on geometrical or surface effects such as the multilamellae mixer, ${ }^{13}$ chaotic flow configuration, ${ }^{14}$ flow recirculation, ${ }^{15}$ or moving droplets. ${ }^{16}$

Parallel lamination micromixers are based on the decrease of the diffusion distance of molecules by splitting the streams to mix into $n$ substreams, and then joining them into one stream as

Laboratoire d'Electrochimie Physique et Analytique (LEPA), Institut des Sciences et Ingénierie Chimiques (ISIC), Ecole Polytechnique Fédérale de Lausanne, CH-1015, Lausanne, Switzerland.E-mail: hubert.girault@epfl. ch; Fax: +41 2169336 67; Tel: +41216933151

$\dagger$ Electronic supplementary information (ESI) available: Finite-element formulation; validation of the finite element convectiondiffusion-reaction model; evaluation of the chemical/diffusional regime; extent to well-known inlet geometries; determination of the Péclet limit; details on the determination of the $\bar{V}_{\mathrm{C}}$ value; contribution of the parabolic flow profile in the performance of the sandwich ABA; evolution of the gain between $\mathrm{ABA}$ and $\mathrm{BAB}$ as a function of the non-dimensional time; relation between the flow rate ratios and the external A layer thickness. See DOI: 10.1039/b815581j alternated laminae of the species to mix. ${ }^{17}$ In small structures, because the time a molecule needs to travel a distance $d$ by diffusion decreases as $1 / d^{2}$, the mixing is quite enhanced. The simplest design is a long microchannel with two inlets $(n=2)$, often called T-mixer or Y-mixer, according to its geometry. The $\mathrm{T}$-mixer has been demonstrated as a tool for the measurements of diffusion coefficients, and as a platform for chemical assays, ${ }^{18-20}$ as a diffusion based immunoassay. ${ }^{21-23}$ Knight et al. introduced a similar laminar flow mixer with three inlets $(n=3)$ known as "hydrodynamic focusing mixing". ${ }^{24}$ The buffer solutions from two symmetric orthogonal side channels focus the sample solution entering from the centre channel into a thin stream. In such a design the sample flow is focused only in the horizontal dimension. Sundararajan et al. presented a generalisation of this conventional two-dimensional (2D) hydrodynamic focusing to a three-dimensional (3D) one that offers the advantage to focus the sample flow in both horizontal and vertical direction to get the sample at the centre of the channel. ${ }^{25}$ More recently, a novel fluid manipulation technique so-called "Microfluidic drifting"26 was presented with two focusing steps combined with a channel curvature resulting in a $3 \mathrm{D}$ hydrodynamically focused flow in the centre of the microfluidic channel. While the 2D and 3D hydrodynamic focusing are based on the focusing of the sample to mix in a thin layer at the microchannel centre, we propose here to study a sandwich mixer design with three flows of equal width. When high throughtput is not the main constraint, this mixerreactor presents the advantage of a simple fabrication process compared to multi-lamellae mixers, and well suited for polymer microchip fabrication technology. In particular, our laboratory is interested in the design of combined post-column reactors and microsprayers for the specific tagging of target molecules after a chromatographic or electrophoretic separation for electrospray mass spectrometry analysis. ${ }^{27,28}$ 
In the field of microfluidics and microreactors, computational methods have proven to be efficient tools to understand physical mechanisms, validate analytical models, design and optimise microsystems. ${ }^{29}$ In the particular case of lamination based mixing, the influence of aspect ratio (heigh/width), asymmetrical vs. symmetrical microstructures or inlet angle has been widely investigated. ${ }^{30-32}$ Finite element simulations have been used in our laboratory to study mixing as in zig-zag structure, ${ }^{15}$ as well as convection-diffusion-reaction phenomena in adsorption immuno-assay, ${ }^{33}$ or multi-tagging of cysteinyl peptides in microchannel during the electrospray ionization process. ${ }^{28}$

In the present study, a sandwich mixer, under pressure-driven flow (PDF) conditions, is investigated to optimise a one-stage chemical reaction $\mathrm{A}+\mathrm{B} \stackrel{k}{\longrightarrow} \mathrm{C}$. In comparison to electroosmotic driven flow (EOF), ${ }^{34}$ pressure-driven flow present some advantages such as the relative ease and flexibility of implementation, insensitivity to surface contamination, ionic strength and $\mathrm{pH}$. Nevertheless, such PDF flows in microfluidic rectangular-shaped channel generate additional complexity in the distribution of analytes because of the velocity profile across one or both cross-sectional dimensions, referred to as Taylor dispersion. ${ }^{18,35-37}$ Herein, we propose two approaches to optimise a chemical reaction in a sandwich mixer. The first one concerns the reactant position according to their diffusive properties taking benefit of the parabolic flow profile. The gain of reaction extent obtained by using three fluid flows instead of two, due to the decrease of the diffusion length, was first quantified. Then, the study was mainly focused on the sandwich design and the optimised positioning of the reactants across the velocity profile, according to their own diffusion coefficient. A comparison with a bulk reaction, where the species are ideally mixed, was done to show the effect of the local residence time value, directly linked to the transversal location of the reactants across the parabolic flow profile. To estimate the contribution of this flow profile, the same simulations were performed with a typical electro-osmotic flat profile. The second approach relies on the decrease of the lateral flow rates as another way to confine the reaction close to the walls while acting on the local kinetics. To extend the conclusions to other chemical reactions, both methods were applied to a first order consecutive reaction $\left(\mathrm{A}+\mathrm{B} \stackrel{k_{1}}{\longrightarrow} \mathrm{AB}_{1}+\mathrm{B} \stackrel{k_{2}}{\longrightarrow} \mathrm{AB}_{2}+\mathrm{B} \stackrel{k_{3}}{\longrightarrow} \mathrm{AB}_{3}\right)$.

\section{Theory and numerical description}

In lamellae mixing, the mixing achievement is depending on the maximal transversal dimension (thickness), $\delta_{h}=2 h / n$, of the $n$ substreams to mix (with $2 h$ the microchannel height). Splitting the flow into $n$ substreams (or layers) allows the decrease of the mixing time. The mean residence time, $\bar{t}_{\mathrm{R}}$, that governs the reaction extent, is fixed by the value of the main channel length $L$ downstream the fluid junction, and the mean linear velocity, $\bar{V}$. At a given time $t$, the species transversal diffusion distance is estimated by $\delta_{i}(t)=\sqrt{D_{i} t}$, where $D_{i}$ is the diffusion coefficient of the species $i$.

Fig. 1A,B depicts the two main geometries investigated: an asymmetrical two-flow geometry (dual mixer) and a symmetrical three-flow design (sandwich mixer). Both geometries present the same channel dimensions $(2 h, L)$ and an inlet angle of $\pm 45^{\circ}$ compared to the main microchannel position. The chemical

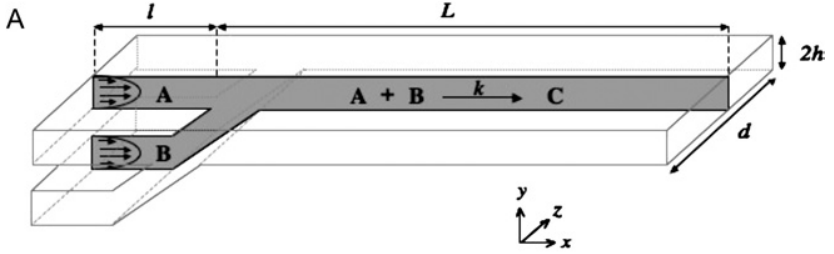

B

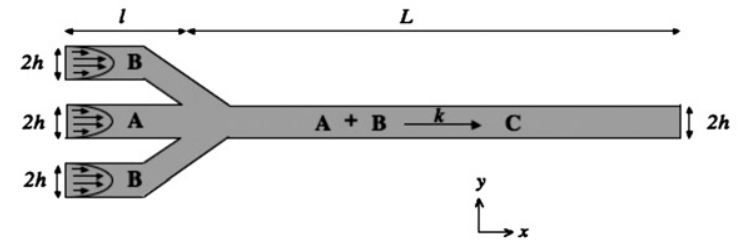

C

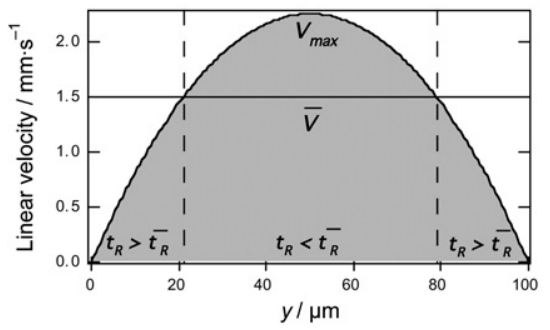

Fig. 1 Geometries with $A$ ) two inlets (dual $A B$ ) and $B$ ) three inlets (sandwich mixer $\mathrm{BAB}$ ). The reactants $\mathrm{A}$ and $\mathrm{B}$ are introduced at the inlets. The three-inlet geometry was also tested with the reactant inverted (sandwich $\mathrm{ABA}$ or $\mathrm{BAB}$ ). A geometry with $L=2 \mathrm{~mm}$ was used for the study of the diffusion coefficient ratio while another one longer with $L=$ $20 \mathrm{~mm}$ was used for the study of the residence time and reaction extent effects. C) Local flow velocity across the microchannel under pressuredriven flow (PDF) condition, with a mean velocity $\bar{V}$ of $1.5 \mathrm{~mm} \mathrm{~s}^{-1}$.

reactants $\mathrm{A}$ and $\mathrm{B}$ are introduced from separated inlets, with the reaction $\mathrm{A}+\mathrm{B} \stackrel{k}{\longrightarrow} \mathrm{C}$ occurring downstream the fluid junction. To insure the comparison of reaction extents between the designs, the incoming flux of the reactants $\mathrm{A}$ and $\mathrm{B}$ are maintained equal (as well as the mean residence time, $\bar{t}_{\mathrm{R}}$ ) by changing the species concentration. Fig. $1 \mathrm{C}$ is the local flow velocity across the microchannel under pressure-driven flow conditions with a mean velocity $\bar{V}$ of $1.5 \mathrm{~mm} \mathrm{~s}^{-1}$. Near the microchannel walls the local residence time is higher than the mean residence time $\left(t_{\mathrm{R}}>\right.$ $\left.\bar{t}_{\mathrm{R}}\right)$ while it is the contrary at the centre of the microchannel $\left(t_{\mathrm{R}}<\right.$ $\bar{t}_{\mathrm{R}}$ ). The main gist of the present study is to take benefits of this local velocity distribution to enhance the reaction extent in a sandwich mixer.

\section{Numerical model and assumptions}

The present model considers the laminar mixing of species along a 2D microchannel section, resulting from the contact between at least two incoming fluids featuring the same dynamic viscosity, $\mu$, and the same density, $\rho$. The following assumptions are proposed:

(i) The solution is assumed to be sufficiently diluted and isothermal so that the viscosity and the density of the fluid can be considered as unmodified by temperature and concentration 
variations. All the simulations consider the injection of two identical fluids (same viscosity and density) as a lot of reactions are performed by mixing the reactants into a same buffer solvent. The diffusion coefficient of each species is also treated as uniform in the entire study domain. (ii) The channel walls are considered to be smooth. (iii) The depth $d$ of the channel is assumed to be much larger than its height $2 h$ ( $2 h$ can be considered as "width" in a design fabrication point of view) so that the velocity gradient in the third dimension can be neglected (2D Cartesian assumption to overcome numerical limitations, valid for $d / 2 h>10$ ). (iv) The fluid is assumed to be Newtonian and its velocity is described according to a parabolic (or Poiseuille) flow profile in laminar conditions. (v) The modification of diffusion laws near the walls is not taken into account. ${ }^{35,36}$

In this model, the mixing process occurring at microscale is studied by solving the momentum and mass transport equations in two steps. First, the Navier-Stokes equation (eqn. 1) and the continuity equation (eqn. 2) are solved in the case of an incompressible fluid in an horizontal channel:

$$
\begin{gathered}
\frac{\partial \boldsymbol{V}^{*}}{\partial t^{*}}+\boldsymbol{V}^{*} \cdot \nabla \boldsymbol{V}^{*}=-\nabla p^{*}+\frac{1}{R e} \nabla^{2} \boldsymbol{V}^{*} \\
\nabla \cdot \boldsymbol{V}^{*}=0
\end{gathered}
$$

where $V^{*}, p^{*}, t^{*}$ are the non-dimensional velocity vector, pressure and time with $V^{*}=V / V_{0}, x^{*}, y^{*}=x, y / L_{0}, p^{*}=p / \rho V_{0}^{2}, t^{*}=$ $t V_{0} / L_{0}$ using the characteristic variables $V_{0}, L_{0}, t_{0}$. For Reynolds numbers (eqn. 4) ranging from $10^{-3}$ to 1 in this study, the NavierStokes equation is solved in laminar conditions without taking into account chaotic instabilities. In addition, we assume that the parabolic flow profile is established at the inlets.

In a second step, the distribution of the species concentration within the geometry is addressed through the convectiondiffusion-reaction equation (eqn. 3 ):

$$
\frac{\partial c_{i}}{\partial t}+\boldsymbol{V}^{*} \cdot \nabla c_{i}^{*}=\frac{1}{P e} \nabla^{2} c_{i}^{*}+R_{i}
$$

where $c_{i}{ }^{*}=c_{i} / c_{0}$ is the concentration normalised by a characteristic concentration $c_{0}$ and $R_{i}$ the rate of consumption of the species $i$. The transversal Reynolds $(R e)$, Péclet $(P e)$ and second Damköler numbers $(D a)^{38-42}$ are defined as following:

$$
\begin{gathered}
R e=\frac{\bar{V} L_{0}}{v} \\
P e_{i}=\frac{\bar{V} L_{0}}{D_{i}} \\
D a_{i}=\frac{k c_{i} \delta_{h}^{2}}{D_{i}}
\end{gathered}
$$

where $\bar{V}$ is the average linear velocity, $L_{0}$ a characteristic transversal length, $\nu$ the kinematic viscosity $(\nu=\mu / \rho), c_{i}$ and $D_{i}$ the volumic concentration and diffusion coefficient of the species $i$, respectively, $k$ the reaction rate constant, and $\delta_{h}$ the thickness of a diffusion sub-layer as described above. The second Damköhler number establishes the ratio between the reaction rate and the mixing rate, in homogeneous solutions (eqn. 6). If mixing is rapid compared to the reaction $(D a \ll 1)$, then the rate of reaction will approach that of a bulk reaction. On the other hand, if mixing is slow $(D a \gg 1)$, then the lamellae will be squashed very slowly on average, and the rate of reaction will approach that of an initially segregated system with no squash. In between $\left(10^{-1}<D a<10^{1}\right)$, the regime is mixed and therefore controlled both by diffusion and kinetics.

\section{Numerical parameters, calibration and normalization}

The finite element formulation (ESI-1 $\dagger^{\dagger}$ ) was implemented in the commercial software Flux-Expert ${ }^{\mathrm{TM}}$ (Astek, Rhône-Alpes, France) on a Mac Pro with Ubuntu Linux 7.10 operating system. The numerical parameters used in this study are given in Table 1. $d$ is not simulated ( $d / 2 h>10$ assumption).

The kinetics and convection-diffusion terms of the model were both validated according to analytical models, ${ }^{27,43,44}$ resulting in a difference of around $1 \%$. More details about the validations are presented in ESI-2.†

The reaction extent $\xi(\%)$ is calculated by normalizing the integrated flux $J_{i}$ of the produced species $(i=\mathrm{C})$ by the flux of the initial reactant $(i=\mathrm{A})$ at the inlet(s) (eqn. 7 and 8$)$.

$$
\begin{aligned}
& J_{i}=\int_{0}^{2 h} \boldsymbol{V}_{i} c_{i} d h \\
& \xi=\frac{J_{\mathrm{C}}}{J_{\mathrm{A}}} \times 100
\end{aligned}
$$

As the flux is depending on the local flow velocity $\boldsymbol{V}_{i}$ the benefits of the near wall region is counterbalanced by its low contribution to the flux (low $\boldsymbol{V}_{i}$ value).

The main part of this study is performed at moderate reaction extents $\left(\xi_{\text {Ref }}<10 \%\right.$ with $k=200 \mathrm{M}^{-1} \mathrm{~s}^{-1}, c_{\mathrm{A}}=c_{\mathrm{B}}=1 \mathrm{mM}, L=$ $2000 \mu \mathrm{m}$ ) for two main reasons. (i) Because our objective was to compare the reaction enhancement between several designs, it appeared more interesting to compare the reaction extent when both design can still gain in performance (i.e. the reaction does not reach completion). According to the $D a$ number, it requires to work in a mixed regime $\left(10^{-1}<D a<10^{1}\right)$. Indeed, the resulting $D a$ number for $k=200 \mathrm{M}^{-1} \mathrm{~s}^{-1}$ is 2 . More details about the diffusional/chemical regimes are presented in ESI-3†. (ii) The reaction rate constant of $210 \mathrm{M}^{-1} \mathrm{~s}^{-1}$ corresponds to the value of the 1,4-benzoquinone, a reactant used for the derivatization of cysteinyl peptides, ${ }^{45}$ as the goal of this numerical work is to optimise a sandwich reactor for the post-column tagging of cysteines. The microchannel dimensions are also the ones of the electrospray emitter microchips used by our group.

Table 1 Numerical reference parameters

\begin{tabular}{llll}
\hline Parameters & $\begin{array}{l}\text { Numerical } \\
\text { value }\end{array}$ & Parameters & Numerical value \\
\hline $2 h / \mu \mathrm{m}$ & 100 & $D_{\mathrm{A}, \mathrm{Ref}} / \mathrm{m}^{2} \mathrm{~s}^{-1}$ & $1 \times 10^{-10}$ \\
$L / \mu \mathrm{m}$ & $2000 / 20000$ & $D_{\mathrm{B}, \mathrm{Ref}} / \mathrm{m}^{2} \mathrm{~s}^{-1}$ & From $1 \times 10^{-10}$ to $110^{-8}$ \\
$l / \mu \mathrm{m}$ & 200 & $k / \mathrm{M}^{-1} \mathrm{~s}^{-1}$ & $\begin{array}{l}20 / 200 / 2000 \\
10^{-6}\end{array}$ \\
$\bar{V} / \mathrm{m} \mathrm{s}^{-1}$ & $1.5 \times 10^{-3}$ & $v / \mathrm{m}^{2} \mathrm{~s}^{-1}$ & \\
\hline
\end{tabular}


Nevertheless, the study is extended to slower $\left(k=20 \mathrm{M}^{-1} \mathrm{~s}^{-1}\right.$, $D a=0.2)$ and faster reaction rates $\left(k=2000 \mathrm{M}^{-1} \mathrm{~s}^{-1}, D a=20\right)$ to determine the applicability of these methods to another reaction kinetics.

Because geometries with orthogonal inlets are often used in the litterature,,$^{3,12,46}$ the performance of the two designs herein presented was compared to an equivalent $\mathrm{Y}$-mixer and cross-mixer. The reaction extent is simulated for identical residence time, reaction rate, reactant concentration and diffusion coefficients. The results are presented in ESI-4. $\dagger$ In all the conditions the relative error is inferior or equal to $0.6 \%$ meaning that the conclusions from this study are also valuable for the most "commonly" used inlet designs such as the Y-mixer, T-mixer or the cross-mixer. According to ESI-5, $\dagger$ the results in this study can be extrapolated (notably in Fig. 4B, 6B and 7 for other values of $(D, t, \delta)$ trio giving the same values of $t_{\mathrm{R}} / t_{\mathrm{D}}$, ), with the condition that, respectively: $P e>50$ for $k=20 \mathrm{M}^{-1} \mathrm{~s}^{-1}, P e>300$ for $k=$ $200 \mathrm{M}^{-1} \mathrm{~s}^{-1}, P e>6000$ for $k=2000 \mathrm{M}^{-1} \mathrm{~s}^{-1}$. Consequently, as this study is performed at $P e=1500$, the results for $k=2000 \mathrm{M}^{-1}$ $\mathrm{s}^{-1}$ cannot be extrapolated.

\section{Results and discussion}

According to the parabolic flow profile occurring in a microchannel, the reaction extent can be enhanced by several ways. The following sections aims to study the influence of the diffusion coefficient ratio $D_{\mathrm{B}} / D_{\mathrm{A}}$, the reaction extent $\xi(k, c, t)$, and the ratio between the diffusion length and the layer thickness $\sqrt{D t} / \delta_{n}$.

\section{Gain due to the number of substreams. $\left(D_{\mathrm{B}} / D_{\mathrm{A}}=1\right)$}

The geometrical advantage of using a three- instead of a two-flow design was first quantified. Fig. 2 represents the reaction extent $\xi$ as a function of the diffusion coefficient ratio of the A and B reactants within the dual and sandwich geometries, for a given reaction rate constant $k=200 \mathrm{M}^{-1} \mathrm{~s}^{-1}$. The reference case for which the reactant diffusion coefficient and incoming flux are equals $\left(D_{\mathrm{A}} / D_{\mathrm{B}}=1\right)$ is first considered (with equal incoming flux $J_{\mathrm{A}}=J_{\mathrm{B}}$ for all the simulations). The reaction extents, $\xi_{\mathrm{Ref}}$, for the dual and sandwich designs are 2.6 and $5.7 \%$, respectively. In addition, two sets of simulations for the sandwich design were performed by inverting the position of the $\mathrm{A}$ and $\mathrm{B}$ reactants at the inlets (ABA or BAB). As $D_{\mathrm{A}}=D_{\mathrm{B}}$ in the reference case, the two sandwich configurations are symmetrical presenting an equal reaction extent, as expected. The gain of the three-flow design compared to the two-flow design (i.e. the ratio $\xi_{\text {sandwich }} /$ $\left.\xi_{\text {dual }}\right)$ is therefore of 2.2 (i.e. $120 \%$ reaction extent improvement) and is due to the decrease of $\delta_{h}$ as it will be illustrated later.

\section{Gain due to the diffusion coefficient ratio of the reactants in a sandwich mixer}

The curves sandwich ABA \& BAB and dual in Fig. 2 illustrate the influence of the A and $B$ reactants position within the inlets, according to their diffusion coefficient values at a reaction rate of $200 \mathrm{M}^{-1} \mathrm{~s}^{-1}$. By increasing the value of the diffusion coefficient of B from $10^{-10}$ to $10^{-8} \mathrm{~m}^{2} \mathrm{~s}^{-1}\left(D_{\mathrm{B}} / D_{\mathrm{A}}\right.$ ranging from 1 to 100$)$, a higher reaction extent is observed when the reactant with the lowest diffusion coefficient (reactant A) is injected from the outer flows, near the microchannel walls where the species get a longer residence time inside the microchannel compared to the mean residence time $\left(t_{\mathrm{R}}>\bar{t}_{\mathrm{R}}\right.$, as shown in Fig. 1C).

Fig. 3 shows the isovalues and the concentration distribution of the species involved in the reaction, along a cross-section at a distance $L / 2=1000 \mu \mathrm{m}$ downstream the fluid junction $\left(D_{\mathrm{B}} / D_{\mathrm{A}}\right.$ $=10$ ). Within the sandwich $\mathrm{BAB}$ geometry (Fig. $3 \mathrm{~A})$, the reactant $\mathrm{B}$ presenting a higher diffusion coefficient, diffuses along all the microchannel cross-section, while the reactant A is mainly located at the microchannel centre. The reaction is therefore mainly focused in the middle of the microchannel where $\mathrm{C}$ is produced, as illustrated on the corresponding isovalues. On the

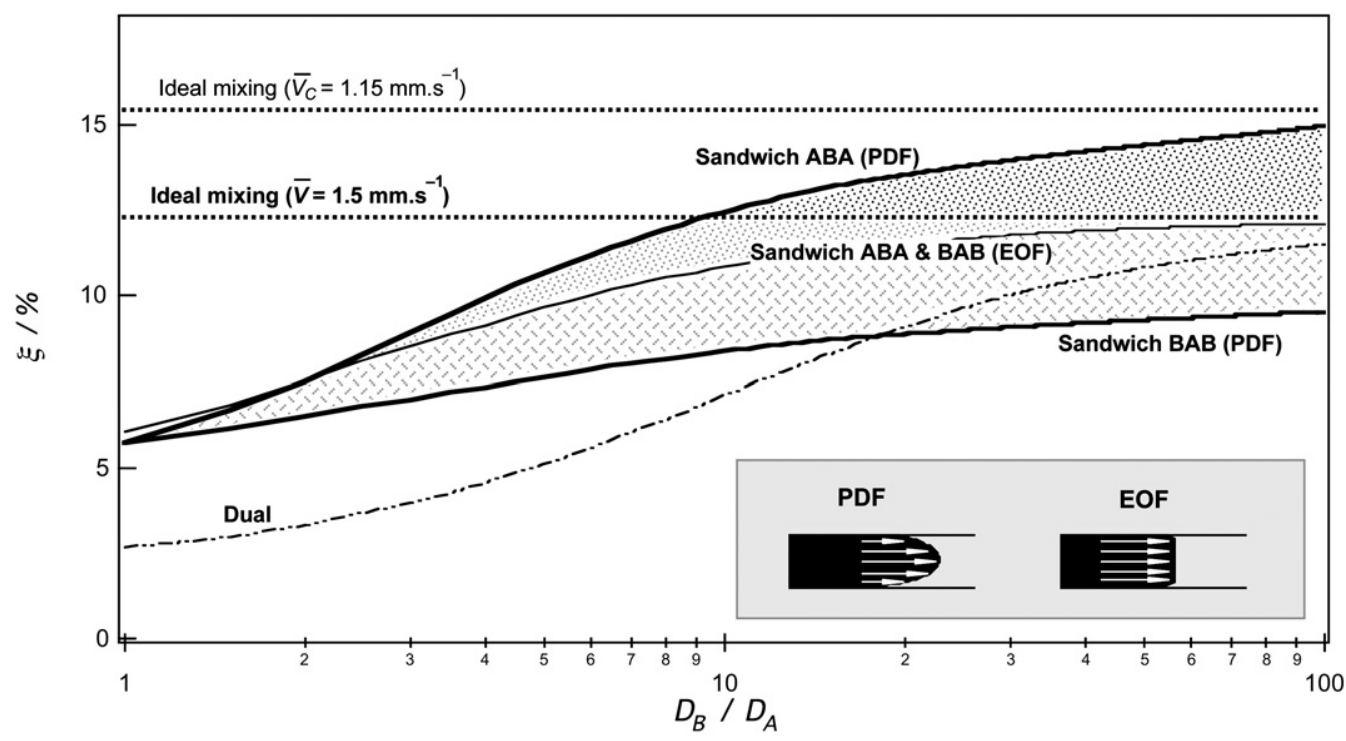

Fig. 2 Effect of the diffusion coefficient ratio of the A and B reactants on the reaction extent within the three geometry configurations (sandwich ABA $\& \mathrm{BAB}$, dual) and comparison with a reaction in bulk condition and with a flat flow profile (EOF). 

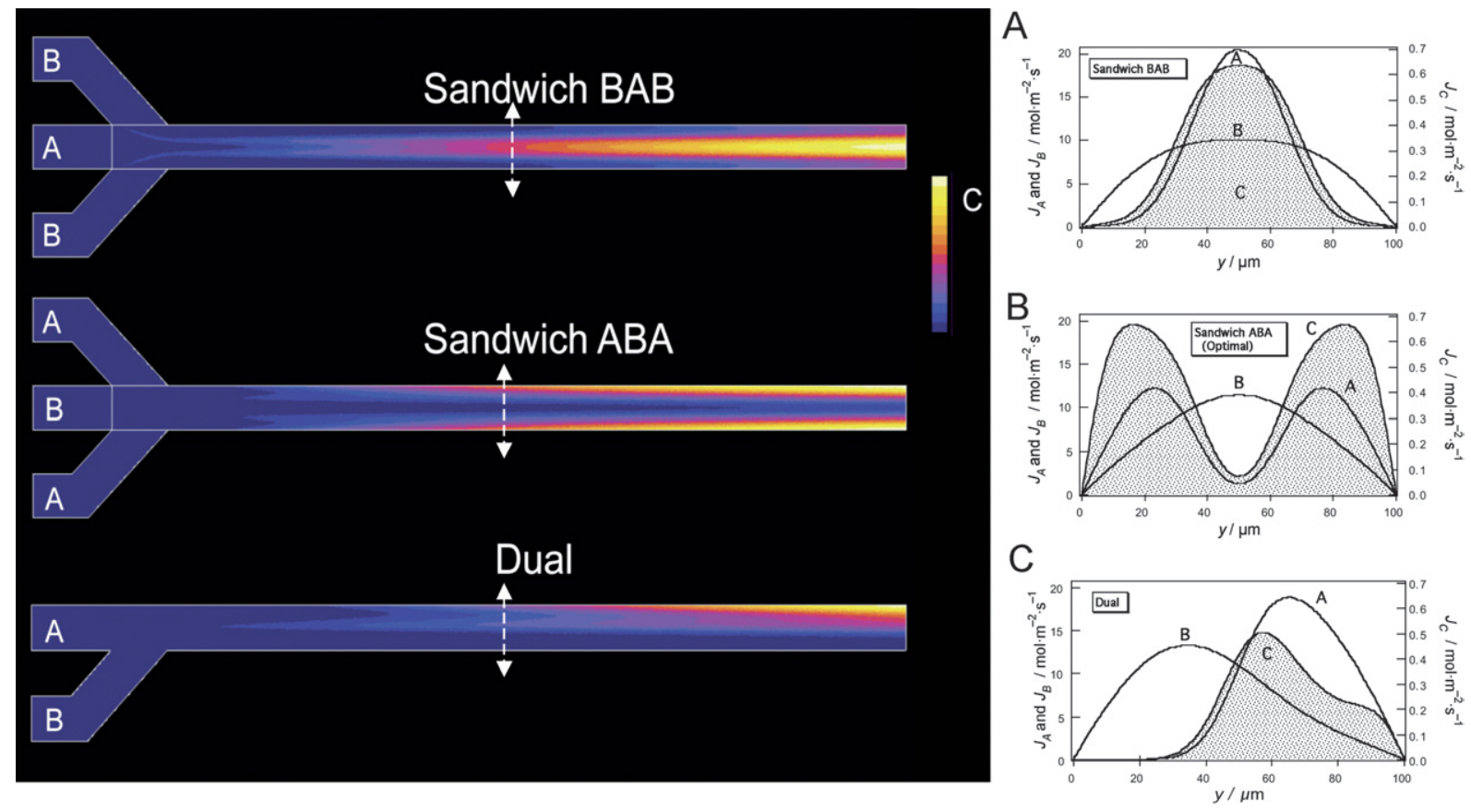

Fig. 3 (Left) Isovalues of the reaction product C along the microchannel length within the three geometries, for $D_{\mathrm{B}} / D_{\mathrm{A}}=10$ and $L=2000 \mu \mathrm{m}$. (Right) Species concentration distribution along the microchannel cross-section, at the position $L / 2$ dowstream of the fluid junction. A,B,C) Species concentration distribution of the reactants $\mathrm{A}$ and $\mathrm{B}$ and products $\mathrm{C}$ with the non-optimal (sandwich $\mathrm{BAB}$ ), optimal (sandwich $\mathrm{ABA}$ ) three-flow or two-flow (dual) geometries, respectively, and for $D_{\mathrm{B}} / D_{\mathrm{A}}=10$. The concentration distribution of $\mathrm{C}$ is represented by the gray surface.

contrary, within the optimal sandwich ABA geometry (Fig. 3B), the reactant $\mathrm{A}$ is close to the walls allowing the reaction to occur where $t_{\mathrm{R}}>\bar{t}_{\mathrm{R}}$. In the case of the dual geometry (Fig. 3C), as the species are initially present in two symmetrical flows, the reaction mainly takes place in one half side of the microchannel. That explains why, for $D_{\mathrm{B}} / D_{\mathrm{A}}>20$, the dual design can provide better results than the non-optimal sandwich $\mathrm{BAB}$.

The reaction extent within the three geometries of mixerreactors (sandwich $\mathrm{ABA} \& \mathrm{BAB}$, dual) was compared to a reaction in bulk conditions where the species are ideally mixed. The simulations were performed in a 1-D microchannel presenting the same dimensions and mean residence time $\bar{t}_{\mathrm{R}}=1.33 \mathrm{~s}(\bar{V}=$ $1.5 \mathrm{~mm} \mathrm{~s}^{-1}$ ). In bulk conditions, the reaction extent is independent of the reactant diffusion coefficients, as illustrated by the constant value of $\xi$ in Fig. 2 (dotted line). By increasing the $D_{\mathrm{B}} /$ $D_{\mathrm{A}}$ ratio, the dual geometry is approaching the reaction extent of an ideal mixing. For $D_{\mathrm{B}} / D_{\mathrm{A}}>10$, as the reaction is mainly occurring next to the walls where the local value $t_{\mathrm{R}}>\bar{t}_{\mathrm{R}}$, the optimal sandwich mixer (ABA) overpasses the bulk reaction extent for a same $\bar{t}_{\mathrm{R}}$. The mean velocity of the product $\bar{V}_{\mathrm{C}}$ is therefore lower than the mean flow velocity $\bar{V}$. When $\bar{V}$ is corrected by the local velocity value of the produced species $C$ across the velocity gradient $\left(\bar{V}_{\mathrm{C}}=1.15 \mathrm{~mm} \mathrm{~s}^{-1}\right.$, see ESI- $\left.6 \dagger\right)$, the reaction extent of the ideal mixing fits to the asymptotic value of the optimal ABA design. Therefore, by taking care of the reactant position, for $D_{\mathrm{B}} / D_{\mathrm{A}}>10$ the optimised sandwich mixer can provide similar or even better results than a corresponding bulk reaction. In addition, to quantify the contribution of the parabolic flow profile in the gain value observed, the reaction extent was compared to simulations performed with a flat electroosmotic driven flow profile within the sandwich geometries. With an EOF profile, the position of the reactants $\mathrm{A}$ and $\mathrm{B}$ do not influence the reaction extent as the flow profile is flat and so, the local residence time along the microchannel cross-section is uniform $\left(t_{\mathrm{R}}=\bar{t}_{\mathrm{R}}, \bar{V}=1.5 \mathrm{~mm} \mathrm{~s}^{-1}\right)$. When increasing the $D_{\mathrm{B}} / D_{\mathrm{A}}$ ratio, the reaction extent with an EOF profile tends to the value of the ideal mixing. Consequently, the curve over-passing observed previously with the optimal ABA design, in PDF conditions, was totally due to the contribution of the parabolic flow profile (quantification vs. $D_{\mathrm{B}} / D_{\mathrm{A}}$ in ESI-7 $\dagger$ ). In optimal conditions, the parabolic profile allows an increase of the reaction extent from 0 for $D_{\mathrm{B}} / D_{\mathrm{A}}=1$ to $22 \%$ for $D_{\mathrm{B}} / D_{\mathrm{A}}=100$. On the contrary, when the position of the reactants is not optimised according to their diffusion coefficients, the loss induced by the parabolic flow profile compared to a flat flow profile reaches $20 \%$ for $D_{\mathrm{B}} / D_{\mathrm{A}}>10$.

To extend the study to other conditions, three different reaction rates $\left(k=20,200\right.$ and $\left.2000 \mathrm{M}^{-1} \mathrm{~s}^{-1}\right)$ were simulated. Fig. 4A summarizes the evolution of the gain calculated between the optimal ABA and non-optimal BAB designs as a function of the diffusion coefficient ratio between $\mathrm{A}$ and $\mathrm{B}$. These values are integrated vertically at a fixed position at the end of a microchannel $L=2000 \mu \mathrm{m}$. With a correct position of the reactant, a gain of $70 \%, 55 \%$ or $20 \%$ reaction enhancement can be reached for $D_{\mathrm{B}} / D_{\mathrm{A}}=100$, and $k=20,200$ and $2000 \mathrm{M}^{-1} \mathrm{~s}^{-1}$, respectively. As expected, a higher gain is obtained for the low reaction rates, as the reaction extent is smaller.

Fig. 4B considers the case where $D_{\mathrm{B}} / D_{\mathrm{A}}=10$ with the gain represented as a function of the non-dimensional time $t_{\mathrm{R}} / t_{\mathrm{D}}\left(D_{\mathrm{B}} /\right.$ $D_{\mathrm{A}}$ ranging from 2 to 100 in ESI- $8 \dagger$ ). It is interesting to note that $t_{\mathrm{R}} / t_{\mathrm{D}}=D t_{\mathrm{R}} / \delta_{\mathrm{n}}^{2}$ also corresponds to the square of the diffusion length normalised by the multilayer thickness $\delta_{\mathrm{n}}$. A longer 


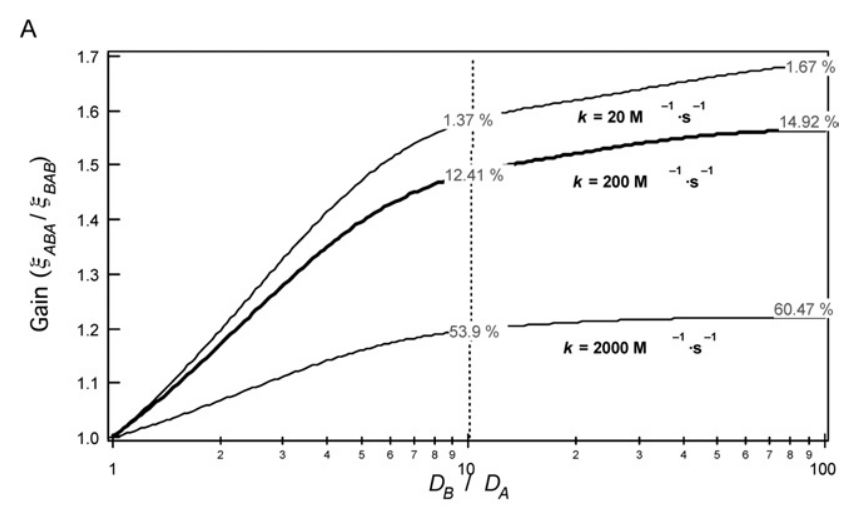

B

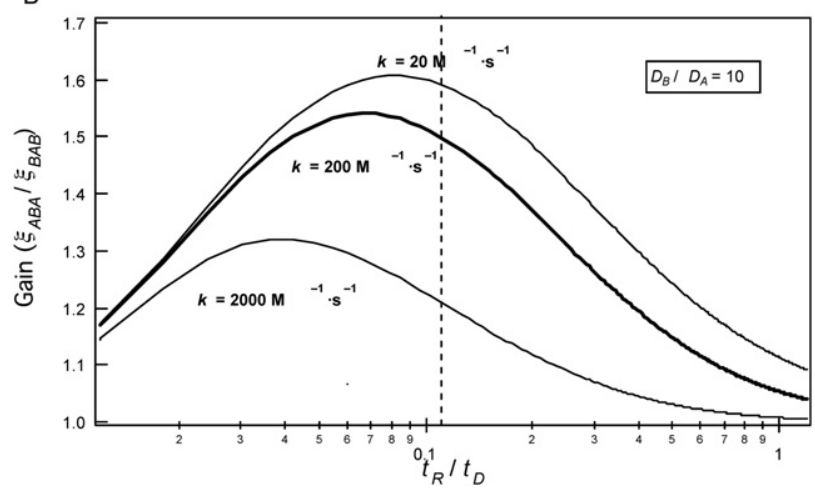

Fig. 4 Effect of the diffusion coefficient ratio. A) Gain of the optimal (ABA) vs. the non-optimal (BAB) sandwich mixer as a function of the reactant diffusion coefficient ratio for $k=20(D a=0.2), 200(D a=2)$ and $2000 \mathrm{M}^{-1} \mathrm{~s}^{-1}(D a=20)$, with $L=2000 \mu \mathrm{m}$. The vertical dashed line corresponds to the $D_{\mathrm{B}} / D_{\mathrm{A}}$ value mainly discussed in the study. B) Gain $\mathrm{ABA} v s$. $\mathrm{BAB}$ as a function of the ratio between the residence time and the diffusion time $\left(t_{\mathrm{R}} / t_{\mathrm{D}}\right)$ for $D_{\mathrm{B}} / D_{\mathrm{A}}=10$ and $L=20000 \mu \mathrm{m}$. The vertical dashed line is the $t_{\mathrm{R}} / t_{\mathrm{D}}$ value of 0.12 that corresponds to $\bar{t}_{\mathrm{R}}=1.33 \mathrm{sec}$ at the end of a microchannel of $L=2000 \mu \mathrm{m}$. microchannel $(L=20000 \mu \mathrm{m})$ was used to enable a sufficient residence time to tend to reaction completion. For low time values, the evolution of the gain follows the same trend for different kinetics with a maximum shifted to higher $t_{\mathrm{R}} / t_{\mathrm{D}}$ for lower reaction kinetics. The increase of the gain up to this maximum is due to the diffusion of the reactant $B$ (with the highest diffusion coefficient) that determines, in a first stage, the transversal location of the reaction across the velocity flow profile. For the optimal ABA geometry, the gain increase is the consequence of the diffusion of $\mathrm{B}$ from the microchannel centre to the walls, confining the reaction in a longer $t_{\mathrm{R}}$ area. When $\mathrm{B}$ is homogeneous along the microchannel cross-section, the slow diffusion of the species A to the centre displaces progressively the position of reaction from the walls to the centre, inducing a loss of the gain. If we consider that the optimisation is of interest for a minimal gain of $20 \%$ reaction enhancement, the position of the reactants in a sandwich mixer is valuable for $t_{\mathrm{R}} / t_{\mathrm{D}}<0.7,0.4$ and 0.1 for $k=20,200$ and $2000 \mathrm{M}^{-1} \mathrm{~s}^{-1}$, respectively.

Fig. 5 represents the evolution of this geometrical gain as a function of the reaction extent calculated in the non-optimal $\mathrm{BAB}$ geometry. The envelop of the gain grouping the three kinetics at a given $D_{\mathrm{B}} / D_{\mathrm{A}}$ clearly shows a decrease of the gain for high reaction extent. For a value above $70 \%$, this optimisation is not useful as the reaction tends to completion, also for the $\mathrm{BAB}$ geometry.

\section{Gain due to the external flow rate $Q_{\mathrm{A}}$ and local kinetics $k c_{\mathrm{A}}$}

Another way to constrain the reaction close to the walls in a sandwich design is to decrease the volumic flow rate $Q_{\mathrm{A}}$ of the outer flows. Fig. 6A illustrates the gain due to this reactant stream dissymmetry by comparison to the reference case (i.e. the ABA design with $Q_{\mathrm{A}, \text { Ref }}=Q_{\mathrm{A}}=Q_{\mathrm{B}}$ and $D_{\mathrm{A}}=D_{\mathrm{B}}$ ). To be in a comparable situation of reactant consumption, the following simulations were performed with an equal flux for the reactants

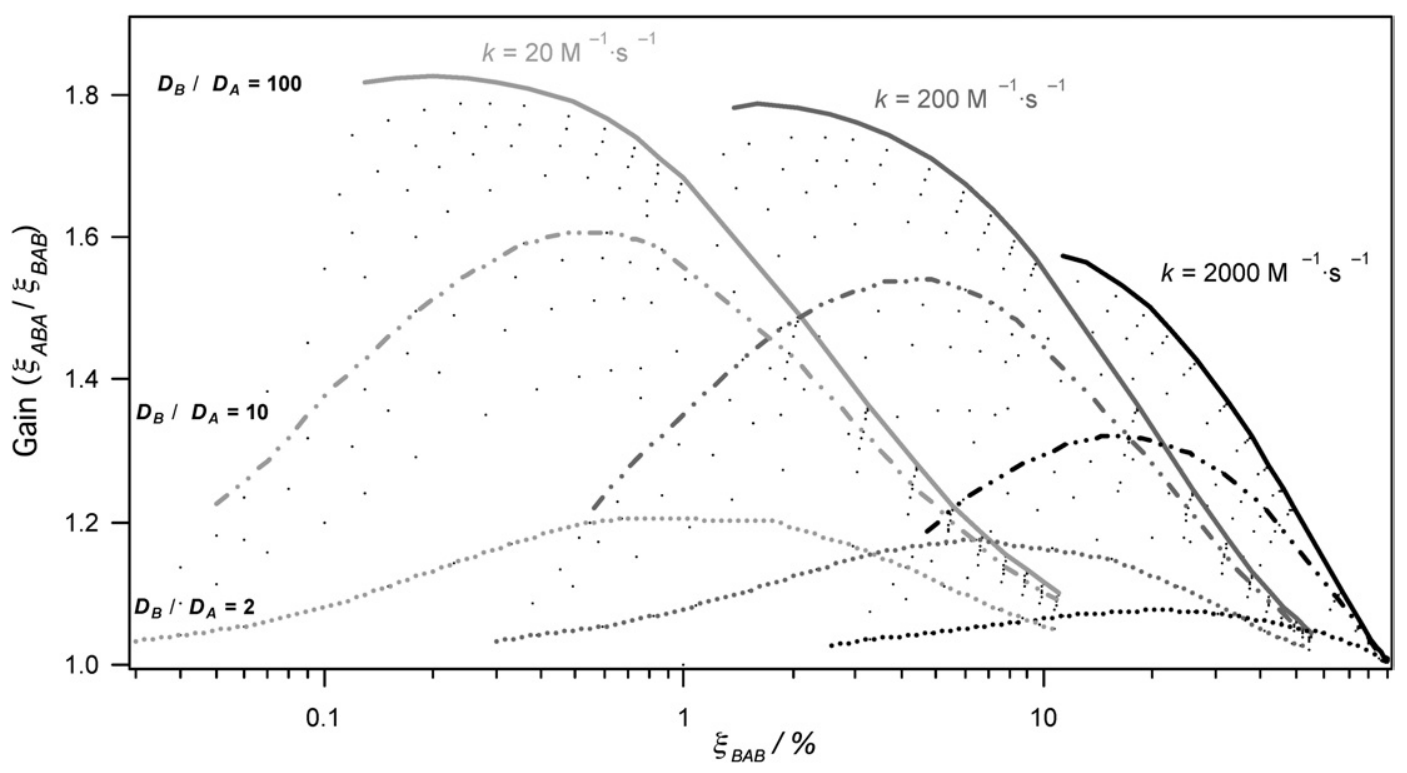

Fig. 5 Gain of the optimal (ABA) vs. non-optimal (BAB) geometry as a function of the reaction extent of the BAB design $(L=2000 \mu \mathrm{m})$, for $k=20,200$ and $2000 \mathrm{M}^{-1} \mathrm{~s}^{-1}$ and $D_{\mathrm{B}} / D_{\mathrm{A}}=2$ (dotted line), 10 (dashed line) and 100 (full line). Dots are the intermediate values for the other $D_{\mathrm{B}} / D_{\mathrm{A}}$. 


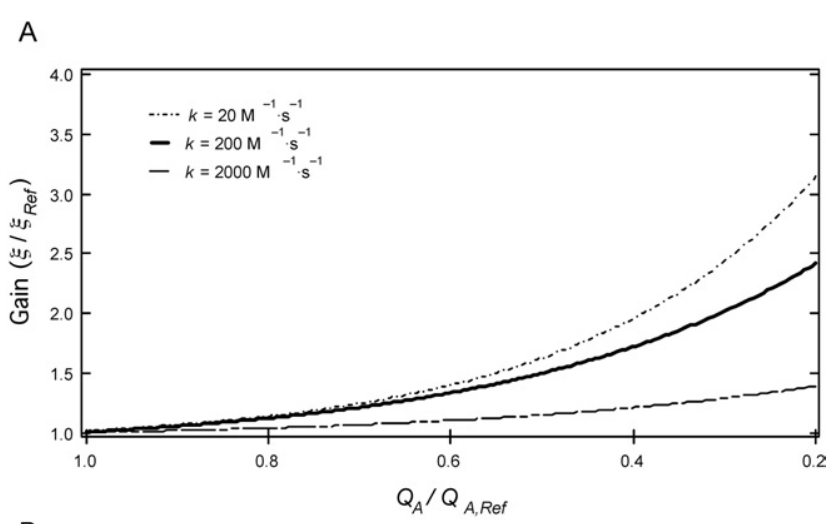

B

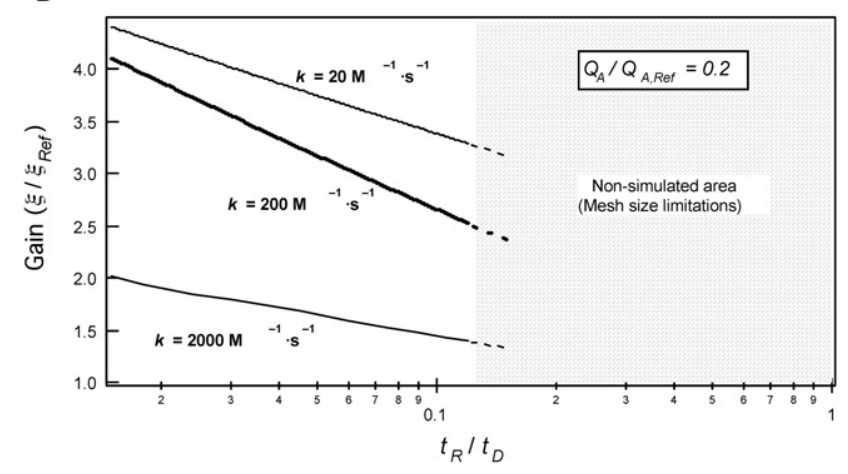

Fig. 6 Effect of the external flow rate decrease. A) Reaction extent gain due to the decrease of the lateral flow rate. The gain is evaluated by comparison with the reference case $\left(Q_{\mathrm{A}, \mathrm{Ref}}=Q_{\mathrm{B}, \mathrm{Ref}}\right)$ at the end of a microchannel length of $L=2000 \mu \mathrm{m}$. All simulation are run with $D_{\mathrm{A}}=$ $D_{\mathrm{B}}$. B) Evaluation of the gain as a function of $t_{\mathrm{R}} / t_{\mathrm{D}}$, with $L=20000 \mu \mathrm{m}$.

A and B. It results in a larger value of the local concentration of A that strongly amplifies the reaction kinetics $\left(k c_{\mathrm{A}} c_{\mathrm{B}}\right.$ in the $R_{i}$ term of eqn. 3) and acts as a "booster" of the reaction starting from the small residence time values. Therefore, the gain observed is both due to this increase of kinetics, the location of the reaction close to the walls, and the decrease of the external flow layer thickness. The results were extracted at corrected positions along the microchannel to maintain the same $\bar{t}_{\mathrm{R}}$ (without this correction, the gain is even stronger). By decreasing $Q_{\mathrm{A}} / Q_{\mathrm{A}, \text { Ref }}$, the gain exponentially increases to reach 2.4 for $Q_{\mathrm{A}} /$ $Q_{\mathrm{A}, \mathrm{Ref}}=0.2$ and $k=200 \mathrm{M}^{-1} \mathrm{~s}^{-1}$ (3.2 and 1.4 for $k=20$ and 2000 $\mathrm{M}^{-1} \mathrm{~s}^{-1}$, respectively). It can be explained by the decrease of the A layer thickness as the $Q_{\mathrm{A}} / Q_{\mathrm{A} \text {, Ref }}$ ratio is reduced. As shown in ESI-9, $\dagger$ this decrease is linear for $0.5<Q_{\mathrm{A}} / Q_{\mathrm{A}, \mathrm{Ref}}<1$ and amplified after $\left(Q_{\mathrm{A}} / Q_{\mathrm{A}, \text { Ref }}<0.5\right)$ which results in the strong gain increase observed in Fig. 6A.

Fig. 6B is the evolution of the gain for $Q_{\mathrm{A}} / Q_{\mathrm{A}, \text { Ref }}=0.2$ as a function of $t_{\mathrm{R}} / t_{\mathrm{D}}$. The maximal gain is reached for small $t_{\mathrm{R}} / t_{\mathrm{D}}$ that corresponds to small residence time and then it decreases progressively. After the fluid junction the reaction is quickly located next to the walls, as $\delta_{\mathrm{A}}$ is decreased by the low lateral flow rate. As for the previous method, the gain decrease is then due to the reaction that is progressively displaced from the walls to the centre with the diffusion of $\mathrm{A}$. The contribution of the parabolic flow profile in the gain value was evaluated by comparing with an EOF profile, and it reaches $11.3 \%$ for the lowest value of the outer flow velocities. It confirms that the kinetic effect is the main source of the present gain increase.

\section{Application to a consecutive reaction}

The former simulations concern the optimisation of a one-stage chemical reaction in a sandwich mixer. In order to extend this study to other kind of chemical reactions, the two methods presented herein were applied to consecutive first order reactions, described as:

$$
\mathrm{A}+\mathrm{B} \stackrel{k_{1}}{\longrightarrow} \mathrm{AB}_{1}+\mathrm{B} \stackrel{k_{2}}{\longrightarrow} \mathrm{AB}_{2}+\mathrm{B} \stackrel{k_{3}}{\longrightarrow} \mathrm{AB}_{3}
$$

where $A$ and $B$ are the reactants and $A B_{1}, A B_{2}$ and $A B_{3}$ the intermediate and final products of the reaction, with $k_{1}=3 k, k_{2}$ $=2 k, k_{3}=k$.

Fig. 7A is an illustration of the first method that consists in placing the reactants in the sandwich mixer-reactor according to their diffusion coefficient ratio $\left(D_{\mathrm{B}} / D_{\mathrm{A}}=10\right.$ and $\left.D_{\mathrm{B}} / D_{\mathrm{A}}=100\right)$. For $D_{\mathrm{B}} / D_{\mathrm{A}}=10$, the one-stage and consecutive reaction are presented. For a consecutive reaction, the gain is 1.5, 2.1 and 3.2 for $A B_{1}, A B_{2}$, and $A B_{3}$ respectively while the reaction extent for a one-stage reaction is around 1.5 . For $D_{\mathrm{B}} / D_{\mathrm{A}}=100$, the gain is even higher and is occurring at lower $t_{\mathrm{R}} / t_{\mathrm{D}}$ as the diffusion of the $\mathrm{B}$ reactant is faster.

Fig. 7B is the comparison of the gain of a one-stage vs. a consecutive reaction when the reaction is located close to the
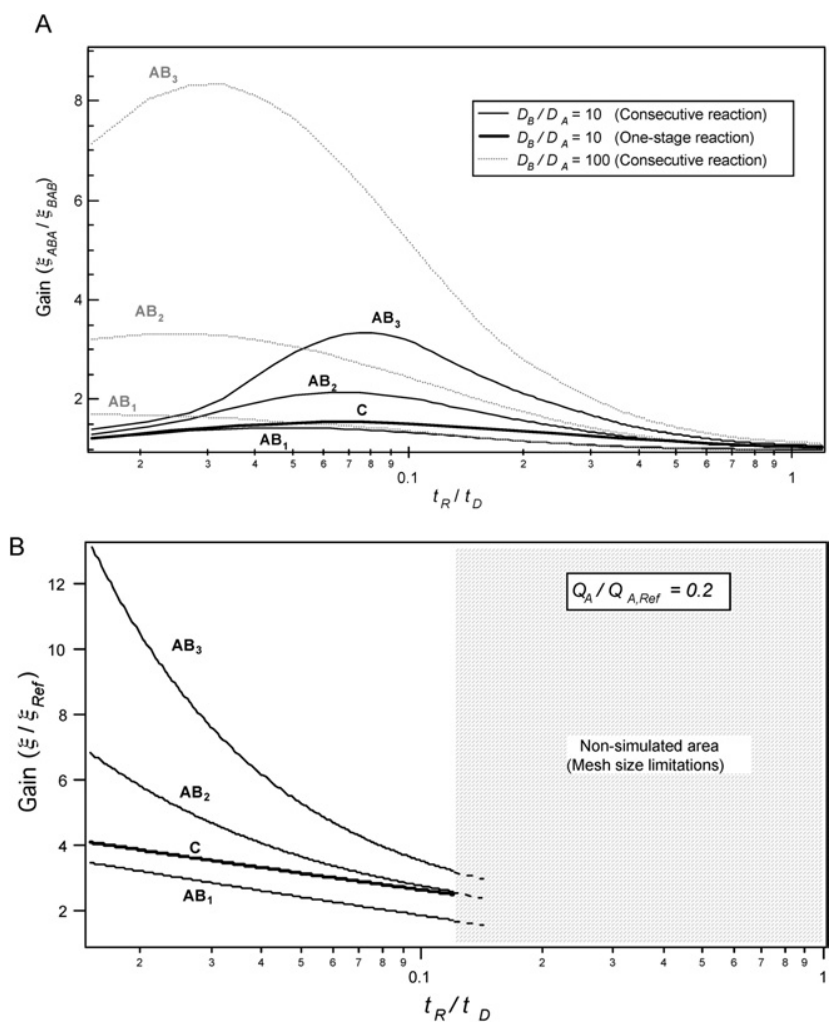

Fig. 7 Consecutive reaction gain vs. the residence time. A) Reaction extent gain by placing the reactants according to their diffusion coefficient ratio and comparison with a one-stage reaction, for $D_{\mathrm{B}} / D_{\mathrm{A}}=10$ and $100(L=2000 \mu \mathrm{m})$. B) Reaction extent gain by decreasing the lateral flow rates (conservation of the flux, $L=20000 \mu \mathrm{m}$ ). 
walls and the kinetics is increased by the higher concentration of A (conservation of the flux), for $Q_{\mathrm{A}} / Q_{\mathrm{A}, \mathrm{Ref}}=0.2$. In this second method and for the same reason as before, the maximal gain is obtained al low $t_{\mathrm{R}} / t_{\mathrm{D}}$, and is 3.5, 6.8 and 13 for $\mathrm{AB}_{1}, \mathrm{AB}_{2}$, and $\mathrm{AB}_{3}$. The maximal gain of the one-stage reaction is 4 .

The present study of chemical reaction optimisation in a sandwich mixer-reactor is even more interesting for consecutive reactions as the reaction is increased for all the intermediate products that finally allow a higher global reaction extent.

\section{Conclusions}

Simulation of chemical reaction extent relying on microfluidic properties and diffusive characteristics of molecules has been investigated in a sandwich mixer by a finite element method.

The diffusive properties ratio of the molecules to react was demonstrated to be an important factor to take into account in such a mixer-reactor. Within this geometry, the reactive species that present the lowest diffusion coefficient have to be introduced from the outer inlets. Thus, the reaction mainly occurs next to the walls where the local residence time is higher than the mean residence time in the microchannel. This optimisation becomes even more relevant as the difference of diffusion coefficient increases, allowing reaching $60 \%$ of reaction enhancement, and is of interest for moderate reaction extent lower than 70\%. Another way to improve the reaction extent in a sandwich mixer is to confine the reaction as close as possible to the walls by the decrease of the outer flow rate. This is a way of increasing the kinetics (high local value of A concentration to keep the flux ratio of $\mathrm{A}$ and $\mathrm{B}$ ) while maintaining a reasonable global flow rate typically required as example for mass spectrometry analysis $\left(V_{\mathrm{B}}\right.$ unchanged). With such a technique, the reaction can be improved by $300 \%$, provided that the over concentration applied from the low velocity outer inlets does not induce a precipitation or adsorption of the molecules. For sure, improvement is no more valuable for advanced residence time (typically $t_{\mathrm{R}} / t_{\mathrm{D}}>0.5$ ) for which the non-optimal case approaches reaction completion. These two methods of optimisation of a sandwich mixer were even more interesting for consecutive reactions as each intermediate species is increased.

For further investigations, it would be interesting to evaluate other kind of reactions as parallel or reversible reactions, as well as second order reactions. In a more general view, the conclusions about the influence of the diffusion coefficient ratio can be applied to different kind of species as, for example, on-line deuterium exchange experiments (as diffusion coefficient of heavy water is $10^{-9} \mathrm{~m}^{2} \mathrm{~s}^{-1}$ compared to proteins at $10^{-10} \mathrm{~m}^{2} \mathrm{~s}^{-1}$ ), on-line chemical modification of peptides/proteins, or antibodyantigen reactions. The study of the influence of the flow rate ratio considers a reaction between species of similar diffusion coefficient that typically can be applied to protein-protein interactions, as example.

In practice, such simulations are very useful to optimise on-line chemical reactions such as, for example, on-line chemical derivatization of biomolecules after a chromatographic or electrophoretic separation where the flow rate of the target molecules is imposed by the separation process. Recently, post-column modification of cysteinyl peptides from a tryptic protein digest was performed directly on an electrospray microchip for mass spectrometry including a mixing unit in order to mix the reactant within the short residence time inside the microchannel. ${ }^{27}$ Instead of implementing a mixing unit to the microchip, the adding of two lateral flows to the device with a correct position of the reactants can be a simple alternative design, requiring that the residence time is not too low for this kind of classical microscopic diffusion based mixing.

\section{Acknowledgements}

Dr. L. Dayon is thanked for his contribution to the analytical model implementation. This work was supported by a Swiss National Science Foundation grant entitled "Development for new analytical tools for proteomics" (Grant No 200020-105489) and a Commission for Technology and Innovation grant entitled "Microfluidic platform for fast immunoassays" (Grant No 9125.1).

\section{References}

1 J. P. Brody, P. Yager, R. E. Goldstein and R. H. Austin, Biophys. J., 1996, 71, 3430-3441.

2 P. S. Dittrich, K. Tachikawa and A. Manz, Analytical Chemistry, 2006, 78, 3887-3907.

3 D. Janasek, J. Franzke and A. Manz, Nature, 2006, 442, 374-380.

4 V. Hessel, H. Lowe and F. Schönfeld, Chem. Eng. Sci., 2005, 60, 2479-2501.

5 N.-T. Nguyen and Z. Wu, J Micromech Microengineering, 2005, 15.

6 T. J. Johnson, D. Ross and L. E. Locascio, Analytical Chemistry, 2002, 74, 45-51.

7 J. L. Lin, K. H. Lee and G. B. Lee, Electrophoresis, 2005, 26, 46054615.

8 A. Dodge, M. C. Jullien, Y. K. Lee, X. Niu, F. Okkels and P. Tabeling, Comptes Rendus Physique, 2004, 5, 557-563.

9 M. H. Oddy, J. G. Santiago and J. C. Mikkelsen, Analytical Chemistry, 2001, 73, 5822-5832.

10 N. Sundaram and D. K. Tafti, Analytical Chemistry, 2004, 76, 37853793.

11 P. Paik, V. K. Pamula and R. B. Fair, Lab Chip, 2003, 3, 253-259.

12 S. Hardt, K. S. Drese, V. Hessel and F. Schönfeld, Microfluidics and Nanofluidics, 2005, 1, 108-118.

13 A. E. Kamholz, B. H. Weigl, B. A. Finlayson and P. Yager, Anal. Chem., 1999, 71, 5340-5347.

14 A. D. Stroock, S. K. W. Dertinger, A. Ajdari, I. Mezic, H. A. Stone and G. M. Whitesides, Science, 2002, 295, 647-651.

15 V. Mengeaud, J. Josserand and H. H. Girault, Analytical Chemistry, 2002, 74, 4279-4286.

16 M. Joanicot and A. Ajdari, Science, 2005, 309, 887-888.

17 S. Hardt, H. Pennemann and F. Schönfeld, Microfluidics and Nanofluidics, 2006, 2, 237-248.

18 A. E. Kamholz, E. A. Schilling and P. Yager, Biophys. J., 2001, 80, 1967-1972.

19 C. D. Costin, R. K. Olund, B. A. Staggemeier, A. K. Torgerson and R. E. Synovec, Journal of Chromatography A, 2003, 1013, 77-91.

20 C. D. Costin and R. E. Synovec, Talanta, 2002, 58, 551-560.

21 A. Hatch, A. E. Kamholz, K. R. Hawkins, M. S. Munson, E. A. Schilling, B. H. Weigl and P. Yager, Nat. Biotechnol., 2001, 19, 461-465.

22 K. E. Nelson, J. O. Foley and P. Yager, Anal. Chem., 2007, 79, 35423548.

23 J. O. Foley, K. E. Nelson, A. Mashadi-Hossein, B. A. Finlayson and P. Yager, Anal. Chem., 2007, 79, 3549-3553.

24 J. B. Knight, A. Vishwanath, J. P. Brody and R. H. Austin, Physical Review Letters, 1998, 80, 3863-3866.

25 N. Sundararajan, M. S. Pio, L. P. Lee and A. A. Berlin, Journal of Microelectromechanical Systems, 2004, 13, 559-567.

26 X. Mao, J. R. Waldeisen and T. J. Huang, Lab Chip, 2007, 7, 12601262.

27 M. Abonnenc, L. Dayon, B. Perruche, N. Lion and H. H. Girault, Analytical Chemistry, 2008, 80, 3372-3378. 
28 L. Dayon, J. Josserand and H. H. Girault, Physical Chemistry Chemical Physics, 2005, 7, 4054-4060.

29 D. A. Boy, F. Gibou and S. Pennathur, Lab Chip, 2008, 8, 1424-1431.

30 N. Aoki, S. Hasebe and K. Mae, AIChE Journal, 2006, 52, 1502-1515.

31 A. Soleymani, E. Kolehmainen and I. Turunen, Chemical Engineering Journal, 2007, 135.

32 J. Yang, X. Pi, L. Zhang, X. Liu, Y. Cao, W. Zhang and X. Zheng, Analytical sciences: the international journal of the Japan Society for Analytical Chemistry, 2007, 23, 697-703.

33 A. Lionello, J. Josserand, H. Jensen and H. H. Girault, Lab Chip, 2005, 5, 1096-1103.

34 U. Tallarek, E. Rapp, T. Scheenen, E. Bayer and H. Van As, Analytical Chemistry, 2000, 72, 2292-2301.

35 R. F. Ismagilov, A. D. Stroock, P. J. A. Kenis, G. Whitesides and H. A. Stone, Applied Physics Letters, 2000, 76, 2376-2378.

36 A. E. Kamholz and P. Yager, Biophysical Journal, 2001, 80, 155160.
37 A. E. Kamholz and P. Yager, Sens Actuators, B Chem, 2002, 82, $117-$ 121.

38 J. M. Ottino, Chemical Engineering Science, 1994, 49, 4005-4027.

39 F. J. Muzzio and J. M. Ottino, Physical Review A, 1990, 42, 58735884.

40 M. J. Clifford, S. M. Cox and E. P. L. Roberts, Chemical Engineering Journal, 1998, 71, 49-56.

41 T. John and I. Mezic, Physics of Fluids, 2007, 19.

42 T. M. Squires, R. J. Messinger and S. R. Manalis, Nature Biotechnology, 2008, 26, 417-426.

43 Z. Wu and N.-T. Nguyen, Microfluid. Nanofluid., 2005, 1, 208-217.

44 L. Dayon, C. Roussel and H. H. Girault, Journal of Proteome Research, 2006, 5, 793-800.

45 L. Dayon, C. Roussel, M. Prudent, N. Lion and H. H. Girault, Electrophoresis, 2005, 26, 238-247.

$46 \mathrm{~N}$. T. Nguyen and Z. Wu, Journal of Micromechanics and Microengineering, 2005, 15. 